\title{
Length of exposure to the hospital environment is more important than antibiotic exposure in healthcare associated infections by methicillin-resistant Staphylococcus aureus: a comparative study
}

\section{Authors}

Ioannis G. Baraboutis ${ }^{1}$ Eleftheria P. Tsagalou ${ }^{2}$ Ilias Papakonstantinou Markos N. Marangos ${ }^{4}$ Charalambos Gogos ${ }^{5}$ Athanasios T. Skoutelis ${ }^{6}$ Haralambos (Harry) Bassaris $^{7}$

Stuart Johnson ${ }^{8}$

${ }^{1} \mathrm{MD}$; Consultant, Infectious Diseases and HIV Division, Evangelismos General Hospital, Athens, Greece ${ }^{2}$ Consultant, Department of Clinical Therapeutics, Alexandra University Hospital, Athens, Greece ${ }^{3}$ Consultant, Internal Medicine, Evangelismos General Hospital, Athens, Greece

${ }^{4}$ Associate Professor

of Infectious Diseases; Consultant, Infectious Diseases, University Hospital of Patras, Greece

${ }^{5}$ Professor of Internal Medicine, University Hospital of Patras, Greece

${ }^{6}$ Professor of Internal

Medicine and Infectious

Diseases; Director,

Infectious Diseases Division,

Evangelismos General Hospital, Athens, Greece

${ }^{7}$ Professor of Internal

Medicine and Infectious

Diseases, University Hospital

of Patras, Greece

${ }^{8}$ Associate Professor of

Infectious Diseases; Loyola

University Medical Center \&

Hines VA Hospital, USA

Submitted on: 02/24/2011 Approved on: 03/16/2011

Correspondence to: Ioannis G. Baraboutis Evangelismos General Hospital

45-47 Ipsilantou Street 10676, Athens, Greece Phone: +30 2107201242 Fax: +30 2107201320 ioannisbaraboutis@yahoo.gr

Conflict of interest: Dr Johnson's work is supported by the VA healthcare system. All the other authors have no financial conflict of interest to declare.

(C)2011 Elsevier Editora Ltda. All rights reserved.

\begin{abstract}
Objectives: Both total antimicrobial use and specific antimicrobials have been implicated as risk factors for healthcare-associated methicillin-resistant Staphylococcus aureus (HCA-MRSA) infection. The aims of this study were: (I) to explore predictors of a new HCA-MRSA infection in comparison with a new healthcare-associated methicillin-sensitive Staphylococcus aureus (HCA-MSSA); (II) to thoroughly assess the role of recent antibiotic use qualitatively and quantitatively. Methods: The time-period for our study was from October 1997 through September 2001. Through applying strict criteria, we identified two groups of inpatients, one with a new HCA-MRSA infection and one with a new HCA-MSSA infection. We recorded demographic, clinical and antibiotic use-related data up to 30 days before the positive culture date. Results: We identified 127 and 70 patients for each group, respectively. Two logistic regression models were carried out to assess the role of antimicrobial use (qualitatively and quantitatively). In model I, duration of hospital stay, presence of chronic wounds, aminoglycoside and fluoroquinolone use retained statistical significance. In model II, duration of hospital stay and history of intubation during the last month stood out as the only significant predictors of a subsequent HCA-MRSA infection. No significant differences in outcome were noted. Conclusions: The length of exposure to the hospital environment may be the best predictor of a new HCA-MRSA infection. Use of aminoglycosides and fluoroquinolones may also stand independently along with presence of chronic ulcers and surgical procedures. No independent association between quantitative antibiotic use and subsequent HCA-MRSA infection was documented.
\end{abstract}

Keywords: methicillin-resistant Staphylococcus aureus; Staphylococcus aureus; antibacterial agents; fluoroquinolones.

\section{INTRODUCTION}

Methicillin-resistant Staphylococcus aureus (MRSA) is considered a pathogen of major importance in healthcare settings. ${ }^{1-3}$ MRSA infections cause significant morbidity and mortality and increased length of hospital stay and cost of care. ${ }^{4-8}$

Risk factors for healthcare-associated MRSA acquisition and infection include age, duration of hospitalization, underlying disease, neonatal or adult ICU or burn unit location, invasive procedures or devices, previous hospitalization, intensity of care, number of inter-ward transfers, coma, low physical function, serum albumin, pressure sores, immunosuppression, proximity to a MRSA-colonized patient, underlying dermatological disease, enteral feedings. ${ }^{4,9-18}$

The issue of antibiotic use as a risk factor is complex and still not completely clarified in full detail. Administration of multiple antibiotics and multiple courses of antibiotics were a significant risk factor for MRSA acquisition and infection from most, but not all, case-control and cohort studies. ${ }^{4,9-23}$ Both total antimicrobial use as well as specific antimicrobial classes have been implicated. ${ }^{11-18,24,25}$ Included in these classes are $\beta$-lactamic antibiotics, ${ }^{14,26-33}$ cephalosporins, ${ }^{11,12,16,22,29}$ aminoglycosides, ${ }^{22,33,34}$ macrolides, ${ }^{27}$ glycopeptides ${ }^{15,27-29,34}$ and fluoroquinolones (FQs). ${ }^{6,11,12,14,26-29,35-37}$

A relatively recently published meta-analysis detected a clear association between exposure to antibiotics and MRSA isolation. Significant risk ratios were found for FQs, glycopeptides, cephalosporins and other $\beta$-lactams. ${ }^{38} \mathrm{~A}$ prediction model, aimed at identifying MRSA bacteremia, predicted that $80 \%$ of the nosocomial bacteremias would be resistant to methicillin if patients had experienced antimicrobials prior to the onset. This model also indicated that there were no differences between antibiotics in the prediction of MRSA. ${ }^{29}$ 
The aims of the study were: (I) to explore predictors of a new healthcare-associated (see definition below) MRSA (HCA-MRSA) infection in comparison with a new healthcare associated MSSA (HCA-MSSA) infection; (II) to thoroughly assess recent antibiotic use (within the last 30 days of the positive culture date) in a qualitative as well as quantitative way as a possibly independent predictor of HCA-MRSA infection; and (III) to especially assess the role of recent FQ use.

\section{MATERIALS AND METHODS}

The Lakeside VA medical center (LSVAMC) was a 280-bed, tertiary-care, urban teaching hospital with approximately 5,000 admissions per year. Computerized medical records for all patients admitted and followed as outpatients to LSVAMC were available from 1991 and thereafter.

Our study protocol was approved by the Northwestern University Institutional Review Board. The time-period for our study was between October 1997 and September 2001.

\section{Inclusion and exclusion criteria}

We retrospectively identified two groups of inpatients, one group with a new positive culture for MRSA and another group with a new positive MSSA culture. Patients with a prior history of any type of positive MRSA or MSSA culture up to 1991 were excluded. We considered the positive culture to be healthcare associated if (I) it was taken more than 48 hours after the current admission date and (II) if it was taken less than 48 hours after the current admission date but the patient had been hospitalized within the last month before the culture date.

Specimen types included in our analysis sent for culture for diagnostic purposes were blood, urine, sputum, wound, fluid and catheter tip specimens. We excluded all patients where SA was originally detected through active surveillance. Additionally, we included only those patients for which there were documented adequate clinical and/or laboratory indications for an active infection (specifically: a urinalysis showing pyuria and urinary symptoms for a urinary tract infection, an adequate sputum specimen with or without a positive Gram stain and respiratory symptoms for a respiratory tract infection, an abnormal body cavity fluid examination for a fluid infection, a clinically infected wound for a wound infection and local signs of inflammation and/or otherwise unexplained fever or hemodynamic instability for positive catheter tip cultures).

Few of the patients included in the study (18 in the MRSA group and 1 in the MSSA group) were outpatients on the day the culture was obtained. We elected not to exclude those patients since all of them had a history of hospitalization the month before the culture date, very likely to have contributed to the acquisition of the organism. There were eight patients for which both a healthcare-associated MSSA infection and a healthcare-associated MRSA infection were identified during the time-period of our study (MSSA was detected first in 8/8). We elected to include those cases in the MSSA group.

We used the computerized medical records of LSVAMC to obtain data pertinent to known risk factors for staphylococcal colonization and infection. We arbitrarily considered events up to 30 days before the positive culture date.

We used the McCabe and Jackson classification system to assess the severity of underlying disease(s) and the Horn severity of illness index for the two groups on the day of the positive culture. ${ }^{39-40}$

\section{Antibiotic data}

Using the electronic pharmacy records of LSVAMC, we were able to obtain detailed data on intravenous antibiotic use (dose strength and number of doses administered) in our two study patient groups during the month before the culture date. To standardize amounts of antimicrobials administered, we calculated defined daily doses (DDD) for all antibiotics. The actual antibiotic DDDs utilized for the purposes of our study are displayed in Table 1.

Table 1. Definitions of defined daily doses (DDDs) of antibiotics studied

\begin{tabular}{lc}
\hline Antibiotic & DDD \\
\hline Penicillin & $8 \mathrm{MU}$ \\
\hline Ampicillin & $4 \mathrm{G}$ \\
\hline Piperacillin & $12 \mathrm{G}$ \\
\hline Ampicillin/sulbactam & $6 \mathrm{G}$ \\
\hline Ticarcillin/clavulanate & $12.4 \mathrm{G}$ \\
\hline Piperacillin/tazobactam & $13.4 \mathrm{G}$ \\
\hline Cefazolin & $3 \mathrm{G}$ \\
\hline Ceftazidime & $4 \mathrm{G}$ \\
\hline Ceftriaxone & $1 \mathrm{G}$ \\
\hline Ceftizoxime & $3 \mathrm{G}$ \\
\hline Aztreonam & $4 \mathrm{G}$ \\
\hline Carbapenem & $2 \mathrm{G}$ \\
\hline Gentamicin & $240 \mathrm{MG}$ \\
\hline Tobramycin & $240 \mathrm{MG}$ \\
\hline Amikacin & $1 \mathrm{G}$ \\
\hline Vancomycin & $2 \mathrm{G}$ \\
\hline Erythromycin & $2 \mathrm{G}$ \\
\hline Metronidazole & $1.5 \mathrm{G}$ \\
\hline Clindamycin & $2.4 \mathrm{G}$ \\
\hline Ciprofloxacin & $800 \mathrm{MG}$ \\
\hline Levofloxacin & $500 \mathrm{MG}$ \\
\hline Trovafloxacin & $300 \mathrm{MG}$ \\
\hline
\end{tabular}


Finally, we collected data about duration of hospital and MSICU and CCU stay for the two study groups until their discharge or death and we compared mortality rates during the current hospital stay.

We used Version 13 of SPSS software for data processing, Student's $t$-test for comparison of continuous variables and Fisher's exact test for categorical variables. Logistic regression analysis (backward stepwise) was used to assess independent predictors of HCA-MRSA infections and Kaplan-Meier analysis for survival comparisons. A p-value of 0.05 or less was used for clinical significance.

\section{RESULTS}

We finally identified 127 patients with a new HCA-MRSA infection and 70 patients with a new HCA-MSSA infection eligible for further analysis. Pertinent demographic, clinical, laboratory and antibiotic usage data of the two groups are depicted on Tables 2 and 3 (characteristics significantly different between the two groups are shown together in Table 2 while those not differing significantly are gathered in Table 3).

\section{Demographic and clinical data}

The mean age of the MRSA group was significantly higher $(\mathrm{p}=0.043)$. All patients were men. A small number of patients in both groups were nursing home residents.

Positive HCA-MSSA blood cultures represented a significantly higher part of the total MSSA cultures than HCA-MRSA (22\% vs. $45.7 \%, \mathrm{p}=0.001$ ) (Table 4 ).

Rates of internal medicine admission and culture ward were significantly higher for the HCA-MSSA group, while the reverse was noted for surgery wards without reaching statistical significance. Additionally, 18 MRSA patients and one MSSA patient were outpatients on the culture date $(p=0.002)$. Overall, only the culture ward distribution was significantly different between the two groups.

\section{Risk factor data}

Patients of the HCA-MRSA group had significantly higher rates of chronic decubitus ulcers while MSSA patients had significantly higher rates of prior hemodialysis. Distribution of McCabe \& Jackson scoring was no different between the two groups.

Patients of the HCA-MRSA group had a significantly longer hospital stay than the HCA-MSSA patients the month before the positive culture $(\mathrm{p}=0.001)$. They also spent significantly more days in the MSICU/CCU $(p=0.036)$ even though the rates of MSICU/CCU stay and interward transfers did not differ between the two groups.

Patients of the HCA-MRSA group had significantly higher rates of surgical procedures, endotracheal intu- bation and presence of indwelling urinary catheter. We found higher rates of "other invasive procedures" in the HCA-MRSA group (see footnote of Table 2) but no differences in the mean number of procedures per patient. Additionally, there were no significant differences in Horn classification at any level of severity.

\section{Antibiotic use}

Overall, significantly more HCA-MRSA patients than HCA-MSSA patients received at least 1, 2 or 3 different antibiotics. On average, HCA-MRSA were characterized by a significantly higher number of different antibiotics used than the MSSA patients $(\mathrm{p}=0.006)$ and significantly higher number of antibiotic-days $(\mathrm{p}=0.005)$. In all subgroups with use of three or more different antibiotics, MRSA patients surpassed MSSA patients. Characteristically, only one MSSA patient received more than five different antibiotics as opposed to 16 MRSA patients (data not shown in table).

In terms of individual antibiotic class use, all statistically significant differences found favored the HCA-MRSA group, including use of penicillins, aminoglycosides, lincosamides and fluoroquinolones. The MSSA group exhibited a non-significant trend for more cephalosporin use.

\section{Quantitative antibiotic use}

Using the method of DDDs, we found significant differences for penicillins, fixed $\beta$-lactam/ $\beta$-lactamase inhibitor combinations, $\beta$-lactamic antibiotics as a whole, aminoglycosides, glycopeptides and fluoroquinolones and overall quantitative antibiotic use.

\section{Multivariate analysis}

Factors and characteristics found to be significantly on univariate analysis were used for logistic regression analysis to assess independent predictors of a new HCA-MRSA infection. Two logistic regression models were carried out to assess the role of antimicrobial usage (Table 5). In the first one (model I) we included qualitative parameters (use versus no use of at least 1, 2 or 3 antibiotics and use versus no use of at least one member of individual antibiotic classes). In the second model (II), we introduced the mean number of different antibiotics used and antibiotic-days per patient, the mean number of Grams of drug administered for significant antibiotic classes and total grams of antibiotics administered per patient. In model I, duration of hospital stay, presence of chronic wounds, aminoglycoside and fluoroquinolones use retained statistical significance, while use of at least two different antibiotics was borderline significant. In model II, duration of hospital stay and history of intubation during the last month stood out as the only significant predictors of a subsequent HCA-MRSA infection. 
Table 2. Univariate analysis of risk factors associated with Staphylococcus aureus infections in the two study groups". Characteristics found to be significantly different between the two groups

\begin{tabular}{|c|c|c|c|c|}
\hline Characteristic & MRSA $(n=127)$ & MSSA $(n=70)$ & OR and 95\% CIs & $\mathbf{p}$ \\
\hline Age (years) & $71.7 \pm 11$ & $68.2 \pm 13$ & N/A & 0.043 \\
\hline Culture ward $\$$ & & & & 0.015 \\
\hline Internal Medicine & $38(29.9)$ & $34(48.6)$ & & \\
\hline Surgery & $47(37.1)$ & $21(30)$ & & \\
\hline MSICU & $12(9.4)$ & $7(10)$ & & \\
\hline $\mathrm{CCU}$ & $12(9.4)$ & $7(10)$ & & \\
\hline Outpatient & $19(14.9)$ & $1(1.4)$ & & \\
\hline \multicolumn{5}{|l|}{ Characteristic } \\
\hline Chronic decubitus ulcers, $\mathrm{n}(\%)$ & $55(43.3)$ & $15(21.4)$ & $2.13(1.33-5.12)$ & 0.003 \\
\hline History of hemodialysis, n (\%) & $5(3.9)$ & 9 (12.9) & $0.28(0.09-0.86)$ & 0.027 \\
\hline \multicolumn{5}{|l|}{ Hospital stay } \\
\hline Days of hospital stay (mean \pm SD) & $18 \pm 9$ & $9 \pm 6.8$ & $1.134(1.086-1.184)$ & 0.001 \\
\hline Days of MSICU/CCU stay (mean \pm SD) & $6.4 \pm 6$ & $3.6 \pm 4.4$ & $1.123(1.002-1.258)$ & 0.046 \\
\hline Other invasive procedure, $\mathrm{n}(\%)^{n}$ & $17(13.4)$ & $2(2.9)$ & $5.25(1.17-23.45)$ & 0.03 \\
\hline Surgery, $\mathrm{n}(\%)^{\|}$ & $44(34.6)$ & $13(18.6)$ & $2.32(1.15-4.70)$ & 0.019 \\
\hline Endotracheal intubation, n (\%) & $48(37.8)$ & $13(18.6)$ & $1.66(1.32-5.71)$ & 0.006 \\
\hline Indwelling urinary catheter $>24$ hours, n (\%) & $105(82.7)$ & $41(58.6)$ & $3.376(1.74-6.54)$ & 0.001 \\
\hline At least 1 antibiotic used & $100(78.7)$ & $40(57.1)$ & $2.78(1.47-5.25)$ & 0.002 \\
\hline At least 2 antibiotics used & $66(51.96)$ & $19(27.1)$ & $2.904(1.55-5.46)$ & 0.001 \\
\hline At least 3 antibiotics used & $46(36.2)$ & $9(12.8)$ & $3.85(1.75-8.46)$ & 0.001 \\
\hline Number of antibiotics used/patient (mean \pm SD) & $2.6 \pm 1.7$ & $1.8 \pm 1.2$ & $1.49(1.10-2.01)$ & 0.009 \\
\hline Antibiotic-days/patient (mean \pm SD) & $5.17 \pm 6.0$ & $1.89 \pm 3.6$ & $1.19(1.08-1.30)$ & 0.005 \\
\hline Penicillins, n (\%) & $65(51.2)$ & $22(31.4)$ & $2.28(1.24-4.22)$ & 0.008 \\
\hline Aminoglycosides, n (\%) & $41(32.3)$ & 9 (12.9) & $3.23(1.46-7.14)$ & 0.004 \\
\hline Lincosamides, n (\%) & $21(16.5)$ & $2(2.9)$ & $6.74(1.53-29.65)$ & 0.012 \\
\hline Fluoroquinolones, n (\%) & $39(30.7)$ & $7(10)$ & $3.99(1.67-9.49)$ & 0.002 \\
\hline \multicolumn{5}{|l|}{$\begin{array}{l}\text { Mean DDDs per patient for antibiotic } \\
\text { classes }(\text { mean } \pm \text { SD) } \ldots\end{array}$} \\
\hline Penicillins & $5.5 \pm 8.1$ & $2.0 \pm 4.5$ & $1.10(1.03-1.17)$ & 0.002 \\
\hline $\begin{array}{l}\text { Fixed } \beta \text {-lactam } / \beta \text {-lactamase } \\
\text { inhibitor combinations }\end{array}$ & $4.5 \pm 7.6$ & $1.9 \pm 4.6$ & $1.08(1.01-1.14)$ & 0.015 \\
\hline$\beta$ - Lactamic antibiotics & $7.3 \pm 9.4$ & $2.5 \pm 4.7$ & $1.11(1.05-1.17)$ & 0.001 \\
\hline Aminoglycosides & $1.9 \pm 4.3$ & $0.4 \pm 1.3$ & $1.31(1.06-1.61)$ & 0.011 \\
\hline Glycopeptides & $5.2 \pm 5.1$ & $0.9 \pm 0.5$ & $5.15(3.31-19.92)$ & 0.017 \\
\hline Fluoroquinolones & $2 \pm 4.3$ & $0.4 \pm 1.5$ & $1.27(1.06-1.51)$ & 0.008 \\
\hline All classes of antibiotics & $25.12 \pm 28.7$ & $7.82 \pm 14.21$ & $1.042(1.02-1.06)$ & 0.001 \\
\hline
\end{tabular}

*All parameters were assessed and measured for the month (last 30 days) before the positive culture date; $\$$ the ward of patient residence at the date of the positive culture; " procedures performed by medicine house staff (bone marrow biopsies, arthrocenteses etc); " includes procedures performed by the surgical team in the operating room and (rarely) at bedside; ${ }^{* *}$ defined daily dose (grams or milligrams of the antibiotic an average person would usually receive in a day, e.g. 2 g for vancomycin). MRSA, methicillin-resistant Staphylococcus aureus; MSSA, methicillin-sensitive Staphylococcus aureus; OR, odds ratio; CI, confidence intervals; N/A, not applicable; MSICU, medical/surgical intensive care unit; CCU, coronary care unit; SD, standard deviation. 
Table 3. Univariate analysis of risk factors associated with Staphylococcus aureus infections in the two study groups". Characteristics found not to be significantly different between the two groups

\begin{tabular}{|c|c|c|c|c|}
\hline Characteristic & MRSA $(n=127)$ & $\operatorname{MSSA}(n=70)$ & OR and 95\% CIs & $\mathbf{p}$ \\
\hline Sex (male/female) & $127 / 0$ & $70 / 0$ & N/A & 1.000 \\
\hline Nursing home resident (n/\%) & $10(7.8)$ & $2(2.8)$ & $0.34(0.073-1.62)$ & 0.18 \\
\hline Admission ward ${ }^{\S}$ & & & & 0.146 \\
\hline Internal Medicine & $42(33.1)$ & $38(54.3)$ & & \\
\hline Surgery & $55(43.3)$ & $22(31.4)$ & & \\
\hline MSICU & $6(4.7)$ & $6(8.6)$ & & \\
\hline $\mathrm{CCU}$ & $4(3.1)$ & $3(4.3)$ & & \\
\hline \multicolumn{5}{|l|}{ Characteristic } \\
\hline Coma $>24$ hours, $\mathrm{n}(\%)$ & $16(12.6)$ & $5(7.1)$ & $1.87(0.65-5.35)$ & 0.24 \\
\hline Diabetes mellitus, n (\%) & $57(44.9)$ & $25(35.7)$ & $1.46(0.80-2.67)$ & 0.21 \\
\hline End-stage renal disease, n (\%) & $7(5.5)$ & $8(11.4)$ & $0.45(0.16-1.3)$ & 0.14 \\
\hline Immunosuppression, n (\%)** & $24(18.9)$ & $15(21.4)$ & $0.85(0.41-1.76)$ & 0.67 \\
\hline $\begin{array}{l}\text { Preexisting permanent central } \\
\text { venous catheter, } \mathrm{n}(\%)\end{array}$ & $13(10.2)$ & $12(17.1)$ & $1.81(0.78-4.23)$ & 0.183 \\
\hline McCabe score & & & & 0.203 \\
\hline NF, n (\%) & 68 (53.3) & 44 (62.9) & & \\
\hline UF, n (\%) & $58(45.7)$ & $24(34.3)$ & & \\
\hline RF, n (\%) & $1(0.8)$ & $2(2.8)$ & & \\
\hline \multicolumn{5}{|l|}{ Hospital stay } \\
\hline Rates of MSICU/CCU stay (\%) & 38.6 & 42.9 & $0.838(0.46-1.51)$ & 0.649 \\
\hline Patient rates with at least 1 interward transfer (\%) & $55.9 \%$ & $50 \%$ & $0.79(0.44-1.42)$ & 0.458 \\
\hline$\geq 2$ transfers & $23.1 \%$ & $22.1 \%$ & $1.064(0.52-2.17)$ & 0.86 \\
\hline$\geq 3$ transfers & $8.7 \%$ & $2.9 \%$ & $3.22(0.69-14.98)$ & 0.135 \\
\hline Paracentesis, n (\%) & $12(9.4)$ & $6(8.6)$ & $1.11(0.40-3.1)$ & 0.838 \\
\hline Bronchoscopy, n (\%) & $3(2.4)$ & $1(1.4)$ & $1.67(0.17-16.36)$ & 0.657 \\
\hline Gastrointestinal endoscopy, n (\%) & $19(15.0)$ & $13(18.6)$ & $0.77(0.35-1.67)$ & 0.511 \\
\hline Angiography, n (\%) & $11(8.7)$ & $3(4.3)$ & $2.12(0.57-7.86)$ & 0.253 \\
\hline $\begin{array}{l}\text { Mean number of procedures } \\
\text { per patient (mean } \pm S D)^{m}\end{array}$ & $0.53 \pm 0.66$ & $0.49 \pm 0.58$ & $1.11(0.69-1.76)$ & 0.32 \\
\hline Mechanical ventilation > 24 hours, $\mathrm{n}(\%)$ & $26(20.5)$ & $9(12.9)$ & $1.74(0.76-3.97)$ & 0.181 \\
\hline Nasogastric tube > 24 hours, n (\%) & $53(41.7)$ & $20(28.6)$ & $1.79(0.95-3.35)$ & 0.067 \\
\hline Enteral feedings > 24 hours, n (\%) & $10(7.9)$ & $7(10)$ & $0.66(0.25-1.76)$ & 0.410 \\
\hline Drainage tube $>24$ hours, n (\%) & $18(14.2)$ & $8(11.4)$ & $1.28(0.53-3.11)$ & 0.586 \\
\hline Presence of central venous catheter in-house, $n$ (\%) & $56(44.1)$ & $24(34.3)$ & $1.51(0.82-2.77)$ & 0.180 \\
\hline Horn classification & & & & 0.581 \\
\hline $1, \mathrm{n}(\%)$ & $4(3.1)$ & $0(0)$ & & \\
\hline $2, \mathrm{n}(\%)$ & $61(48)$ & $29(41.4)$ & & \\
\hline $3, \mathrm{n}(\%)$ & $47(37)$ & $34(48.6)$ & & \\
\hline $4, \mathrm{n}(\%)$ & $15(11.8)$ & $7(10)$ & & \\
\hline Cephalosporins, n (\%) & $18(14.2)$ & $17(24.3)$ & $0.55(0.26-1.14)$ & 0.108 \\
\hline
\end{tabular}


Table 3. Univariate analysis of risk factors associated with Staphylococcus aureus infections in the two study groups*. Characteristics found not to be significantly different between the two groups (Cont.)

\begin{tabular}{|c|c|c|c|c|}
\hline Characteristic & $\operatorname{MRSA}(n=127)$ & MSSA $(n=70)$ & OR and $95 \%$ CIs & $\mathbf{p}$ \\
\hline Fixed $\beta$-lactam/ $\beta$-lactamase inhibitor combinations, n (\%) & $43(33.9)$ & $17(24.3)$ & $1.59(0.82-3.08)$ & 0.162 \\
\hline$\beta$-Lactamic antibiotics, $\mathrm{n}(\%)$ & $80(63)$ & $36(51.4)$ & $1.61(0.89-2.90)$ & 0.115 \\
\hline Glycopeptides, n (\%) & $32(25.2)$ & $11(15.7)$ & $1.81(0.85-3.85)$ & 0.123 \\
\hline Macrolides, n (\%) & $67(5.5)$ & $1(1.4)$ & $4.02(0.48-33.40)$ & 0.197 \\
\hline Nitroimidazoles, n (\%) & $7(5.5)$ & $0(0.0)$ & -- & 0.99 \\
\hline \multicolumn{5}{|l|}{ Mean DDDs per patient for antibiotic classes (mean \pm SD) \|\|} \\
\hline Cephalosporins & $1.0 \pm 3.2$ & $0.3 \pm 0.9$ & $1.15(0.97-1.38)$ & 0.111 \\
\hline Macrolides & $3.3 \pm 3$ & 1 & $1.72(0.43-6.99)$ & 0.445 \\
\hline Lincosamides & $4.9 \pm 4$ & $5.8 \pm 3.4$ & $0.95(0.66-1.35)$ & 0.765 \\
\hline Nitroimidazoles & $7.7 \pm 5.7$ & 0 & -- & 0.99 \\
\hline
\end{tabular}

*All parameters were assessed and measured for the month (last 30 days) before the positive culture date; § the initial admission ward during the hospitalization where the new infection was noted; * corticosteroid use of at least 7.5 mg prednisone daily or equivalent, cytotoxic chemotherapy or other potent immunosuppressive agents, whole-body radiation or presence of human immunodeficiency virus infection; ${ }^{11}$ mean number of procedures: the sum of paracenteses, bronchoscopies, gastrointestinal endoscopies, angiographies, hemodialysis sessions, central line placement during current admission and other invasive procedures (hemodialysis sessions were counted as 1 procedure); "| || defined daily dose.

MRSA, methicillin-resistant Staphylococcus aureus; MSSA, methicillin-sensitive Staphylococcus aureus; OR, odds ratio; CI, confidence intervals; N/A, not applicable; MSICU, medical/surgical intensive care unit; CCU, coronary care unit; NF, non-fatal illness; UF, ultimately fatal; RF, rapidly fatal; SD, standard deviation.

Table 4. Distribution of culture specimens in the two study groups

\begin{tabular}{lccc}
\hline Type of culture specimen & MRSA & MSSA & p \\
\hline Blood, n (\%) & $28(22)$ & $32(45.7)$ & 0.001 \\
\hline Urine, n (\%) & $26(20.5)$ & $8(11.4)$ & 0.119 \\
\hline Sputum, n (\%) & $23(18.1)$ & $9(12.9)$ & 0.421 \\
\hline Other fluid, n (\%) & $6(4.7)$ & $8(11.4)$ & 0.090 \\
\hline Wound drainage fluid, n (\%) & $45(35.4)$ & $16(22.9)$ & 0.078 \\
\hline CVC tip, n(\%) & $3(2.4)$ & $4(5.7)$ & 0.248 \\
\hline
\end{tabular}

MRSA, methicillin-resistant Staphylococcus aureus; MSSA, methicillin-sensitive Staphylococcus aureus; CVC, central venous catheter. Note that cumulative numbers and percentages of culture specimens may exceed the number of patients and 100 respectively, since some patients had positive cultures from more than one site on the culture date.

Table 5. Predictors of healthcare associated methicillin-resistant Staphylococcus aureus infections by logistic regression analysis [backward stepwise (Wald)]

\begin{tabular}{lccc}
\hline Model & Variable & OR \& 95\% CI & p \\
\hline & Duration of hospital stay & $1.163(1.059-1.278)$ & 0.002 \\
I (qualitative indices & Chronic ulcers & $0.203(0.044-0.944)$ & 0.042 \\
of antibiotic exposure only) & Aminoglycosides & $11.123(1.70-72.73)$ & 0.012 \\
& Fluroquinolones & $7.74(1.26-47.32)$ & 0.027 \\
\hline $\begin{array}{l}\text { II (quantitative indices } \\
\text { of antibiotic exposure only) }\end{array}$ & (Use of at least 2 different antibiotics) & {$[0.168(0.027-1.038)]$} & $(0.055)$ \\
\hline
\end{tabular}


Table 6. Outcome analysis for the two study groups

\begin{tabular}{lccc}
\hline Hospital stay (after the positive culture date) & MRSA (n= 127) & MSSA (n= 70) & $\mathbf{p}$ \\
\hline Duration of hospital stay (days) & $21 \pm 21$ & $19 \pm 20$ & 0.467 \\
\hline MSICU/CCU stay, n,\% & $23(18.1)$ & $19(27.1)$ & 0.138 \\
\hline Duration of MSICU/CCU stay (days) & $31 \pm 26 \pm 21$ & 0.081 \\
\hline Mortality during current hospital stay, n(\%) & $26(20.5)$ & $14(20)$ & 0.937 \\
\hline
\end{tabular}

MRSA, methicillin-resistant Staphylococcus aureus; MSSA, methicillin-sensitive Staphylococcus aureus; MSICU, medical-surgical intensive care unit; CCU, coronary care unit.

\section{Analysis of outcomes}

We performed comparative analysis of several outcome indicators between the two study groups (Table 6). A relatively higher percentage of MSSA patients spent time in the MSICU/CCU after the positive culture date but the MRSA group showed a trend for more days of MSICU/CCU stay on average. No difference in survival was observed (Figure 1).

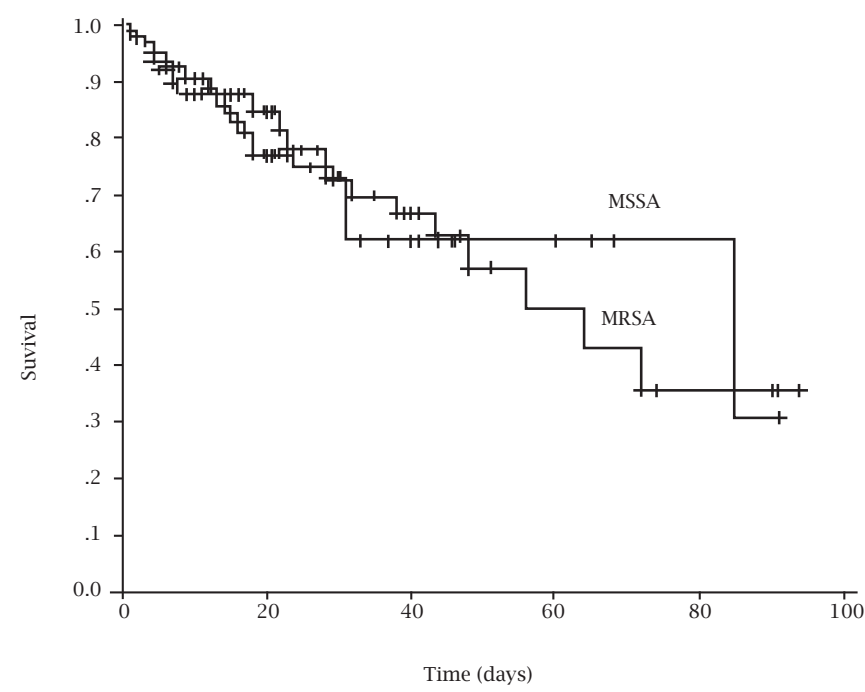

Figure 1: Comparative analysis of survival during current admission between the two groups.

MRSA, methicillin-resistant Staphylococcus aureus; MSSA, methicillin-sensitive Staphylococcus aureus.

\section{DISCUSSION}

We were able to demonstrate that, in our study population, significant predictors of a new HCA-MRSA infection as opposed to a new HCA-MSSA infection were the duration of recent hospital stay, presence of chronic wounds, history of intubation and use of any aminoglycoside and fluoroquinolone.
The findings of our study are in significant agreement with similar studies. However, there are, in our opinion, some interesting features arisen from our analysis that worth further discussion.

In our study, MRSA patients were older than the MSSA patients, which has been the case in some but not all studies. ${ }^{9,27,28}$ Additionally, the MRSA group seemed to be different from the MSSA group in certain parameters. The MRSA group consisted of a preponderance of "surgical" patients while the MSSA group of mostly "internal medicine" patients. Accordingly, the MRSA patients had significantly higher rates of surgery during the current admission and also higher rates of endotracheal intubation but not prolonged mechanical ventilation, indicating elective intubation for surgery. The MRSA group had clearly spent more days in-hospital and more days in the MSICU/CCU before the culture date, a difference frequently found in similar studies. ${ }^{9,28}$ Additionally, the MRSA patients had higher rates of decubitus ulcers and presence of indwelling urinary catheter for more than 24 hours, both known risk factors for MRSA colonization and infection. ${ }^{28}$ On the other hand, the MSSA group included a higher percentage of chronic hemodialysis patients.

\section{Antibiotic use}

Overall, the MRSA group was characterized by significantly heavier antibiotic use than the MSSA group by all types of analyses we performed. The mean number of antibiotics used per patient differed significantly, while the mean antibioticdays were at least two-fold for the MRSA group.

By the two types of logistic regression analysis we performed, we were able to show that recent exposure, in a qualitative way, to aminoglycosides and fluoroquinolones stood out as independent indicators of a subsequent HCA-MRSA infection. These results are in agreement with prior studies, looking at qualitative use of those antibiotics. ${ }^{6,11,12,14,22,26-29,33-38}$ Additionally, use of at least two different antibiotics during the previous month was at the border of statistical significance. The underlying mechanisms have not been completely clarified but several lines of evidence exist. Fluoroquinolones, for example, are excreted in apocrine and eccrine sweat of healthy individuals 
undergoing antibiotic therapy and could promote the acquisition of MRSA by eradicating susceptible microorganisms such as MSSA ${ }^{41}$ Subinhibitory concentrations of ciprofloxacin induced the production of fibronectin-binding proteins and increased the adhesion to fibronectin-coated surfaces in vitro, promoting host colonization. ${ }^{42}$ Fluoroquinolones might influence oxacillin resistance by selective inhibition or by killing of the more susceptible subpopulations in heteroresistant S. aureus. ${ }^{43}$ In our study, 120/127 (94.49\%) and 122/127 (96.1\%) of the MRSA strains had intermediate sensitivity or frank resistance to at least one member of the fluoroquinolone and aminoglycoside classes respectively, while the respective numbers for the MSSA group were $32 / 70$ (45.71\%) and 44/70 (62.85\%) (data not shown in table).

A lesser number of studies has evaluated the role of antibiotics quantitatively. ${ }^{25,28,44}$ In our study, even though quantitative use of several antibiotic classes and antibiotics as a whole was much heavier in the MRSA group, no quantitative index emerged as independent predictor of HCA-MRSA infections. Graffunder et al showed that quantitative use of levofloxacin was independently associated with MRSA infection. ${ }^{27}$ Ernst et al, using a case-case-control study design, found significant qualitative and quantitative differences in fluoroquinolone use between the MRSA and MSSA bacteremia groups but not between the MRSA and the uninfected group. ${ }^{44}$ In our study, the role of antibiotics was surpassed by the effect of length of exposure to the hospital environment and surgical procedures, as represented by the history of intubation. These two factors, especially the former, are, without any doubt, among the strongest predictors of MRSA infections in older and newer studies. ${ }^{4,9-18,20-23,25-28,33,34}$ Obviously, differences in our results compared to the above-described studies may be partly due to different study design and differences in populations studied, as noted in a recent meta-analysis. ${ }^{38}$ Additionally, from a subanalysis of the blood isolates of our study groups, the mean number of antibiotic-days did emerge as independent predictor of a subsequent HCA-MRSA bacteremia. ${ }^{45}$

\section{Outcome analysis}

No significant differences in morbidity or mortality were found (Table 6). Studies especially focusing on morbidity differences are limited, while the impact of methicillin resistance on mortality has been extensively studied for SA bacteremia and several- but not all- studies, including two meta-analyses, have reported a real effect. ${ }^{27,46-49} \mathrm{~A}$ main reason for this apparent disagreement may be a case-mix effect due to the variety of SA infections included in our study groups and this attenuation phenomenon has also been noted elsewhere. ${ }^{18}$ Another reason may be the significantly higher percentage of bloodstream infections in the MSSA group.

\section{Strengths and limitations of the study}

We used strict inclusion and exclusion criteria to create our study groups, empowered by the major degree of completeness of our data. The LSVAMC population, form which the study groups derived, was largely homogeneous (exclusively males with similar patterns of comorbidities) and also relatively stable in the sense that those patients would seldom be admitted in another institution and, if so, this would be one out of the other VA hospitals of the Chicago area. Since the computerized filing system was common to all Chicago VA hospitals, such information was readily available. The same held true for outpatient visits. The strict criteria used may be more important for the HCA-MSSA group. Rates of methicillin-resistance in hospitalized patients are increasing worldwide, so that creating this specific kind of patient groups is becoming more and more difficult.

Despite the existence of complete records about intravenous antibiotics for inpatients and outpatients, that was not the case with inpatient or outpatient oral antibiotics. Nevertheless, it is safe to say, at least in regards to inpatient oral antibiotic therapy, that the volume of the oral therapy was relatively small compared to its intravenous counterpart for the great majority of hospitalized patients at the LSVAMC, even for antibiotics with excellent oral bioavailability like the fluoroquinolones.

Additionally, we designed this study electing to "look back" at the last 30 days before the positive culture date, this way creating a fixed period of exposure to risk factors (or time at risk) for the two groups. Previous studies of HCA-MRSA infections may have not adjusted enough for differences in time at risk.

Besides qualitative information about antibiotic use, we also had accurate quantitative data in our disposition. The fact that quantitative indices of antibiotic use did not emerge as independent predictor of a subsequent HCA-MRSA infection does not abrogate the clear-cut heavier antibiotic exposure of the MRSA group. This observation along with the preponderance of "surgical" patients in the MRSA group may have immediate real-life implications. The use of antimicrobial agents in hospitals in the US and several European countries is often excessive or unnecessary. ${ }^{50}$ The problem may be augmented in surgical subspecialty clinics, where antibiotic "coverage" may be used to provide a sense of safety and awareness of adverse consequences of prolonged use and efforts for rational use are less well established.

There were several limitations in our study. We did not include a third uninfected group for comparison purposes ${ }^{28,44}$ Even though such a control group has frequently not been included in similar studies, it would certainly have made our observations more widely valid. Due to inadequate data, we were not able to calculate and compare device-days (catheter-days, etc). Finally, hospital cost data about the two groups were not available.

\section{CONCLUSION}

Length of exposure to the hospital environment may be the best predictor of a new HCA-MRSA infection. Use of aminoglycosides and fluoroquinolones may also stand independently along with presence of chronic ulcers 
and exposure to surgical procedures. No independent association between quantitative antibiotic use with the method of DDDs and subsequent HCA-MRSA infection was documented. Efforts for minimized and well-targeted patient stay in-hospital along with rational use of antibiotics by physicians of all subspecialties represent both immediate needs and important indices of quality of medical care.

\section{REFERENCES}

1. Horan TC, White JW, Jarvis WR et al. Nosocomial infection surveillance, 1984. MMWR 1986; 35: 17SS-29SS.

2. Preheim LC, Rimland D, Bittner MJ. Methicillin-resistant Staphylococcus aureus in Veterans Administration Medical Centers. Infect Control 1987 May; 8(5): 191-4.

3. Wakefield DS, Pfaller M, Massanari RM et al. Variation in methicillin-resistant Staphylococcus aureus occurrence by geographic location and hospital characteristics. Infect Control 1987 Apr; 8(4): 151-7.

4. Onorato M, Borucki MJ, Baillargeon G et al. Risk factors for colonization or infection due to methicillin-resistant Staphylococcus aureus in HIV-positive patients: a retrospective case-control study. Infect Control Hosp Epidemiol 1999 Jan;20(1): 26-30.

5. Crane LR, Levine DP, Zervos MJ, Cummings G. Bacteremia in narcotic addicts at the Detroit Medical Center. I. Microbiology, epidemiology, risk factors and empiric therapy. Rev Infect Dis 1986 May-June; 8(3): 364-73.

6. Campillo B, Dypeyron C, Richardet JP. Epidemiology of hospital-acquired infections in cirrhotic patients: effect of carriage of methicillin-resistant Staphylococcus aureus and influence of previous antibiotic therapy and norfloxacin prophylaxis. Epidemiol Infect $2001 \mathrm{Dec} ; 127(3)$ : 443-50.

7. Herwaldt LA. Control of methicillin-resistant Staphylococcus aureus in the hospital setting. Am J Med 1999 May; 106 (5A): $11 \mathrm{~S}-18 \mathrm{~S}$.

8. Abramson MA, Sexton DJ. Nosocomial methicillin-resistant and methicillin-susceptible Staphylococcus aureus primary bacteremia: at what costs? Infect Control Hosp Epidemiol 1999 Jun; 20(6): 408-11.

9. Sadoyama G, Filho PPG. Risk factors for methicillin-resistant and sensitive Staphylococcus aureus in a Brazilian University hospital. Braz J Infect Dis 2000 Jun; 4(3): 135-43.

10. O' Sullivan NP, Keane CT. Risk factors for colonization with methicillin-resistant Staphylococcus aureus among nursing home residents. J Hosp Infect 2000 Jul; 45(3): 206-10.

11. Asensio A, Guerrero A, Quereda C, Lizan M, Martinez-Ferrer $\mathrm{M}$. Colonisation and infection with methicillin-resistant Staphylococcus aureus: Associated factors and eradication. Infect Control Hosp Epidemiol 1996 Jan; 17(1): 20-8.

12. Crowcroft N, Maguire H, Fleming M, Peacock J, Thomas J. Methicillin-resistant Staphylococcus aureus: Investigation of a hospital outbreak using a case-control study. J Hosp Infect 1996 Dec; 34(4): 301-9.

13. Law MR, Gill ON. Hospital-acquired infection with methicillinresistant and methicillin-sensitive staphylococci. Epidemiol Infect 1988 Dec; 101(3): 623-9.

14. Dziekan G, Hahn A, Thune K et al. Methicillin-resistant Staphylococcus aureus in a teaching hospital: Investigation of nosocomial transmission using a matched case-control study. J Hosp Infect 2000 Dec; 46(4): 263-70.
15. Coll PP, Crabtree BF, O'Conner PJ. Clinical risk factors for methicillin-resistant Staphylococcus aureus bacteriuria in a skilled-care nursing home. Arch Fam Med 1994 Apr; 3(4): 357-60.

16. Washio M, Mizoue T, Kajioka T et al. Risk factors for methicillin-resistant Staphylococcus aureus (MRSA) infection in a Japanese elderly care nursing home. Public Health 1997 May; 111(3): 187-90.

17. Warshawsky B, Hussain Z, Gregson DB et al. Hospital- and community-based surveillance of methicillin-resistant Staphylococcus aureus: Previous hospitalization is the major risk factor. Infect Control Hosp Epidemiol 2000 Nov; 21911): 724-7.

18. Hershow RC, Khayr WE, Smith NL. A comparison of clinical virulence of nosocomially acquired methicillin-resistant and methicillin-sensitive Staphylococcus aureus infections in a University hospital. Infect Control Hosp Epidemiol 1992 Oct; 13(10): 587-93.

19. Santoro-Lopes G, de Gouvea EF, Monteiro RC et al. Colonization with methicillin-resistant Staphylococcus aureus after liver transplantation. Liver Transpl 2005 Feb; 11(2): 203-9.

20. Yee-Lean L, Gupta G, Cesario T et al. Colonisation by Staphylococcus aureus resistant to methicillin and ciprofloxacin during 20 months' surveillance in a private skilled nursing facility. Infect Control Hosp Epidemiol 1996 Oct; 17(10): 649-53.

21. Boyce JM, Landry M, Deetz TR, DuPont HL. Epidemiologic studies of an outbreak of nosocomial methicillin-resistant Staphylococcus aureus infections. Infect Control 1981 MarApr; 2(2) : 110-6.

22. Peacock JE, Marsik FJ, Wenzel RP. Methicillin-resistant Staphylococcus aureus : Introduction and spread within a hospital Ann Intern Med 1980 Oct; 93(4): 526-32.

23. Crossley K, Loesch D, Landesman B et al. An outbreak of infections caused by strains of Staphylococcus aureus resistant to methicillin and aminoglycosides. I. Epidemiologic studies. J Infect Dis 1979 Mar;139(3): 280-7.

24. Thompson RL, Cabezudo I, Wenzel RP. Epidemiology of nosocomial infections caused by methicillin-resistant Staphylococcus aureus. Ann Intern Med 1982 Sep; 97(3): 309-17.

25. Harbarth S, Rutschmann O, Sudre P, Pittet D. Impact of methicillin resistance on the outcome of patients with bacteremia caused by Staphylococcus aureus. Arch Intern Med 1998 Jan; 158(2): 182-9.

26. Hori S, Sunley R, Tami A, Grundmann H. The Nottingham Staphylococcus aureus population study: prevalence of MRSA among the elderly in a university hospital. J Hosp Infect 2002 Jan; 50(1):25-9.

27. Graffunder EM, Venezia RA. Risk factors associated with nosocomial methicillin-resistant Staphylococcus aureus (MRSA) infection including previous use of antimicrobials. J Antimicrob Chemother 2002 Jun; 49(6): 999-1005.

28. Weber SG, Gold HS, Hooper DC, Karchmer AW, Carmeli Y. Fluoroquinolones and the risk for methicillin-resistant Staphylococcus aureus in hospitalized patients. Emerg Infect Dis 2003 Nov; 9(11): 1415-22.

29. Lodise TP, Jr, McKinnon PS, Rybak M. Prediction model to identify patients with Staphylococcus aureus bacteremia at risk for methicillin resistance. Infect Control Hosp Epidemiol 2003 Sep; 24(9): 655-61.

30. Baggett HC, Hennessy TW, Rudolph K et al. Community-onset methicillin-resistant Staphylococcus aureus associated with antibiotic use and the cytotoxin Panton-Valentine leukocidin during a furunculosis outbreak in rural Alaska. J Infect Dis 2004 May 1; 189(9): 1565-73. 
31. Garcia-Vazquez E, Gomez J, Banos R et al. A comparative study of patients with methicillin susceptible versus methicillin resistant Staphylococcus aureus bacteremia: epidemiology and prognostic factors. Med Clin (Barc) 2007 May 12; 128(18): 681-6.

32. Tumbarello M, de Gaetano Donati K, Tacconelli E et al. Risk factors and predictors of mortality of methicillin-resistant Staphylococcus aureus (MRSA) bacteraemia in HIV-infected patients. J Antimicrob Chemother 2002 Sep; 50(3): 375-82.

33. Ward TT, Winn RE, Hartstein AL, Sewell DL. Observations relating to an inter-hospital outbreak of methicillin-resistant Staphylococcus aureus: Role of antimicrobial therapy in infection control. Infect Control 1981 Nov-Dec; 2(6): 453-9.

34. Asensio A, Guerrero A, Quereda C, Lizan M, Martinez-Ferrer M. Colonisation and infection with methicillin-resistant Staphylococcus aureus: Associated factors and eradication. Infect Control Hosp Epidemiol 1996 Jan; 17(1): 20-8.

35. Tacconelli E, Venkataraman L, De Girolami PC, D’Agata EM. Methicillin-resistant Staphylococcus aureus bacteraemia diagnosed at hospital admission: distinguishing between community-acquired versus healthcare-associated strains. J Antimicrob Chemother 2004 Mar; 53(3): 474-9.

36. Santoro-Lopes G, de Gouvea EF, Monteiro RC et al. Colonization with methicillin-resistant Staphylococcus aureus after liver transplantation. Liver Transpl 2005 Feb; 11(2): 203-9.

37. Zahar JR, Clec'h C, Tafflet $M$ et al. Is methicillin resistance associated with a worse prognosis in Staphylococcus aureus ventilator-associated pneumonia? Clin Infect Dis 2005 Nov 1; 41(9): 1224-31.

38. Tacconelli E, De Angelis G, Cataldo MA, Pozzi E, Cauda R. Does antibiotic exposure increases the risk of methicillin-resistant Staphylococcus aureus (MRSA) isolation? A systematic review and meta-analysis. J Antimicrob Chemother 2008: 61: 26-38.

39. McCabe WR, Jackson GG. Gram-negative bacteremia (I. Etiology and ecology). Arch Intern Med 1962 Dec; 110: 83-91.

40. Mc Cabe WR, Jackson GG. Gram-negative bacteremia (II. Clinical, laboratory and therapeutic observations). Arch Intern Med 1962 Dec; 110: 92-100.
41. Hoiby N, Jarlov JO, Kemp M et al. Excretion of ciprofloxacin in sweat and multiresistant Staphylococcus epidermidis. Lancet 1997 Jan 18; 349(9046):167-9.

42. Bisognano C, Vaudaux PE, Lew DP, Ng EY, Hooper DC. Increased expression of fibronectin-binding proteins by fluoroquinolone-resistant Staphylococcus aureus exposed to subinhibitory levels of ciprofloxacin. Antimicrob Agents Chemother 1997 May; 41(5): 906-13.

43. Venezia RA, Domaracki BE, Evans AM, Preston KE, Graffunder EM. Selection of high-level oxacillin resistance in heteroresistant Staphylococcus aureus by fluoroquinolone exposure. J Antimicrob Chemother 2001 Sep; 48(3): 375-81.

44. Ernst EJ, Raley G, Herwaldt LA, Diekema DJ. Importance of control group selection for evaluating antimicrobial use as a risk factor for methicillin-resistant Staphylococcus aureus bacteremia. Infect Control Hosp Epidemiol 2005 Jul; 26(7): 634-7.

45. Baraboutis IG, Tsagalou EP, Skoutelis AT et al. Predictors of methicillin resistance in healthcare-associated Staphylococcus aureus bloodstream infections: the role of recent antibiotic use. J Chemother 2010 Dec;22(6):424-7.

46. Soriano A, Martinez JA, Mensa J et al. Pathogenic significance of methicillin resistance for patients with Staphylococcus aureus bacteremia. Clin Infect Dis 2000 Feb; 30(2): 368-73.

47. Topeli A, Unal S, Akalin HE. Risk factors influencing clinical outcome in Staphylococcus aureus bacteraemia in a Turkish University Hospital. Int J Antimicrob Agents 2000 Feb; 14(1): 57-63.

48. Cosgrove SE, Sakoulas G, Perencevich EN, Schwaber MJ, Karchmer AW, Carmeli Y. Comparison of mortality associated with methicillin-resistant and methicillin-susceptible Staphylococcus aureus bacteremia: a meta-analysis. Clin Infect Dis 2003 Mar 1;36(5):592-8. Epub 2003 Feb 7.

49. Whitby M, McLaws ML, Berry G. Risk of death from methicillin-resistant Staphylococcus aureus bacteraemia: a meta-analysis. Med J Aust 2001 Sep 3;175(5):264-7.

50. Muto CA, Jernigan JA, Ostrowsky BE et al. SHEA guideline for preventing nosocomial transmission of multidrug-resistant strains of Staphylococcus aureus and enterococcus. Infect Control Hosp Epidemiol 2003 May; 24(5): 362-86. 